ALLERGY AND ASTHMA

\section{What 'drives' IL-4 versus IL-13 signalling?}

\begin{abstract}
Interleukin-4 (IL-4) and $\underline{\mathrm{IL}-13}$ are crucially involved in the development of allergic responses through their induction of T helper $2\left(\mathrm{~T}_{\mathrm{H}} 2\right)$ cells and promotion of IgE production. Both of these cytokines use the common $\gamma$-chain $\left(\gamma_{c}\right)$-related IL-4 receptor $\alpha$-chain (IL-4R $\alpha$ ) to signal through three cytokine-receptor combinations: IL-4 signals through the type I receptor IL-4R $\alpha-\gamma_{c}$, and both IL-4 and IL-13 can signal through the type II receptor IL-4R $\alpha-$ IL-13R $\alpha 1$. This study describes the crystal structures of these three ligand-receptor combinations, which provide insights into
\end{abstract}

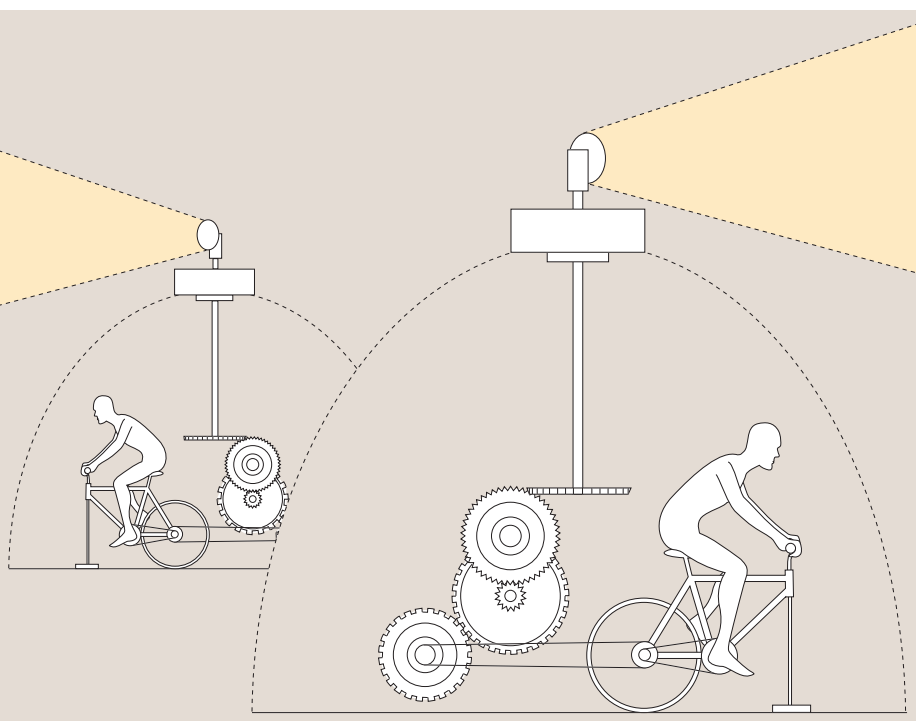

the mechanisms and consequences of receptor degeneracy versus specificity.

The overall structures of IL-4 and IL-13 and their receptors were similar to the canonical architecture that has been resolved for other cytokine-receptor complexes, with the exception of an extra aminoterminal immunoglobulin-like domain (D1) in IL-13R $\alpha 1$ that forms sheet-like contacts with the dorsal surfaces of both IL-4 and IL-13 in the type II receptor. A hydrophobic patch on the surface of IL-13, but not IL-4, forms an additional contact with an apposing hydrophobic patch on D1 of IL-13R $\alpha 1$; this might explain why D1 is known to be required for binding and signalling by IL- 13 but not IL- 4 .

The authors also looked at the thermodynamics of complex assembly using soluble receptor extracellular domains. Whereas IL- 4 binds first to IL-4R $\alpha$ (the 'driver') followed by recruitment of either $\gamma_{c}$ or IL-13R $\alpha 1$ (the 'triggers'), IL-13 binds first to IL-13R $\alpha 1$ (the 'driver') followed by recruitment of IL-4R $\alpha$ (the 'trigger'). Two charged residues in IL-4 that are crucial for its interaction with IL- $4 \mathrm{R} \alpha$ are similarly present in IL-13, but the IL-13-IL-4R $\alpha$ interface lacks other interactions around the charged residues that were observed at the IL-4-IL-4R $\alpha$ interface. This explains why IL-13 must first recruit IL-13R $\alpha 1$ to strengthen the interaction with IL-4R $\alpha$. The IL-13-IL-13R $\alpha 1$ driver has a high affinity for the IL-4R $\alpha$ trigger compared with the low affinity of the IL-4-IL-4R $\alpha$ driver for either $\gamma_{c}$ or IL-13R $\alpha 1$ triggers. The IL-13 receptor complex is therefore more stable than the IL-4 receptor complexes, and the authors suggest that recruitment of the triggers is energetically limiting for IL-4 complexes, which could affect the different signalling properties of IL-4 and IL-13.

Both cytokines induce phosphorylation of signal transducer and activator of transcription 6 ( $\underline{\text { STAT6 })}$, but in human cell lines, IL-4 could stimulate STAT6 phosphorylation at significantly lower doses than IL-13, and the response to IL-13 was markedly slower. This would seem to contradict the thermodynamic data showing that recruitment of the trigger receptor IL-4R $\alpha$ in IL-13 signalling is more energetically favourable than recruitment of the converse trigger receptor IL-13R $\alpha 1$ in IL-4 signalling. However, this could be explained if the number of IL- $4 \mathrm{R} \alpha$ chains was limiting compared with the number of IL-13R $\alpha 1$ chains, which seems to be the case in the cell lines that were analysed.

So, the structural and kinetic data provide insights into how the same type II heterodimer (IL-4R $\alpha$ IL-13R $\alpha 1$ ) can respond to different ligands (IL-4 and IL-13) with different signalling potencies and kinetics.

Kirsty Minton

ORIGINAL RESEARCH PAPER LaPorte, S. L. et al. Molecular and structural basis of cytokine receptor pleiotropy in the interleukin-4/13 system. Cell 132, 259-272 (2008) 